\title{
ON THE LOWER BOUND FOR A CLASS OF HARMONIC FUNCTIONS IN THE HALF SPACE*
}

\author{
Zhang Yanhui (张艳慧) \\ Department of Mathematics, Beijing Technology and Business University, Beijing 100048, China \\ E-mail: zhangyanhui@th.btbu.edu.cn \\ Deng Guantie (邓冠铁) \\ School of Mathematical Sciences, Beijing Normal University, Beijing 100875, China \\ E-mail: denggt@bnu.edu.cn \\ Kou Kit Ian (高洁欣) \\ Department of Mathematics, Faculty of Science and Technology, University of Macau, China \\ E-mail: kikou@umac.mo
}

\begin{abstract}
The main objective is to derive a lower bound from an upper one for harmonic functions in the half space, which extends a result of B. Y. Levin from dimension 2 to dimension $n \geq 2$. To this end, we first generalize the Carleman's formula for harmonic functions in the half plane to higher dimensional half space, and then establish a Nevanlinna's representation for harmonic functions in the half sphere by using Hörmander's theorem.

Key words harmonic function; Carleman's formula; Nevanlinna's representation for half sphere; lower bound
\end{abstract}

2010 MR Subject Classification 31B05; 35J05

\section{Introduction and Main Results}

A version of the well-know Liouville theorem states that if a real harmonic function $u(x)\left(x \in \mathbf{R}^{n}\right)$ has a finite upper bound, then $u(x)$ is a constant in $\mathbf{R}^{n}$. In this paper, we derive an estimate of lower bound of harmonic functions from its upper bound in the upper half space. This estimate is useful and important for studying harmonic functions and the growth properties because its associated assumption is weaker than that of the Maximum Principle.

The method to derive an estimate of a lower bound for a harmonic function from its upper bound in a half plane is using Carleman's formula and Nevanlinna's representation in the halfdisk. This motivates that to obtain a similar estimate in a higher dimension, one might need to

*Received September 10, 2010; revised October 26, 2011. Project supported by the Academic Human Resources Development in Institutions of Higher Learning under the Jurisdiction of Beijing Municipality (IHLB 201008257) and Scientific Research Common Program of Beijing Municipal Commission of Education (KM 200810011005) and PHR (IHLB 201102) and research grant of University of Macau MYRG142(Y1-L2)-FST111KKI.

${ }^{\dagger}$ Corresponding author: Deng Guantie. 
first generalize Carleman's formula from the half plane to the half space in $\mathbf{R}^{n}$ and Nevanlinna's representation from the half disk to the half sphere in $\mathbf{R}^{n}$ for a harmonic function. We know that Carleman's formula for harmonic functions in half plane is derived by taking the real part of contour integral for analytic functions. Taking the real part of the contour integral is impossible in half space of $\mathbf{R}^{n}$, but it is possible for us to use the contour integral to construct Green's function of half sphere in $\mathbf{R}^{n}$ by Hörmander's method. In this paper, Carleman's formula for a harmonic function in the half space is established by Green's function, Navanlinna's representation in the half sphere is obtained by using Hörmander's theorem, and the estimate of lower bound from an upper one in the half space is obtained by using Carleman's formula and Navanlinna's representation in the half sphere. For the proof, we let $\mathbf{R}^{n}$ denote the $n$ dimensional Euclidean space and $|x|$ denote the Euclidean norm. Set $x=\left(x_{1}, \cdots, x_{n-1}, x_{n}\right)=$ $\left(x^{\prime}, x_{n}\right)$ with $x^{\prime}=\left(x_{1}, \cdots, x_{n-1}\right)$. Then $\left|x^{\prime}\right|^{2}=x_{1}^{2}+\cdots+x_{n-1}^{2},|x|^{2}=\left|x^{\prime}\right|^{2}+x_{n}^{2}$. For simplicity, a point $x^{\prime}$ in $\mathbf{R}^{n-1}$ is often identified with $\left(x^{\prime}, 0\right)$ in $\mathbf{R}^{n}$. The boundary and closure of a subset $\Omega$ of $\mathbf{R}^{n}$ are denoted by $\partial \Omega$ and $\bar{\Omega}$ respectively. Let $\Omega^{+}=\left\{x=\left(x^{\prime}, x_{n}\right): x_{n}>0, x \in \Omega\right\}$. We also write $B(r)$ and $S(r)$ for the open ball and the sphere of radius $r(>0)$ and centered at the origin, and $B(r, R)=B(R) \backslash \bar{B}(r)(R>r>0)$.

In the sense of Lebesgue measure $\mathrm{d} x^{\prime}=\mathrm{d} x_{1} \cdots \mathrm{d} x_{n-1}, \mathrm{~d} x=\mathrm{d} x^{\prime} \mathrm{d} x_{n}$. The mirror image of $x=\left(x^{\prime}, x_{n}\right)$ in the hyperplane $\mathbf{R}^{n-1} \times\{0\}=\mathbf{R}^{n-1}$ is denoted by $x^{*}=\left(x^{\prime},-x_{n}\right)$.

A twice continuously differentiable function $u(x)$ defined on $\Omega^{+}$is harmonic if $\Delta u \equiv 0$, where $\triangle=\partial_{1}^{2}+\partial_{2}^{2}+\cdots+\partial_{n}^{2}$ with $\partial_{j}^{2}$ denote the second partial derivative with respect to the $j$ th coordinate variable $(j=1, \cdots, n)$.

The following theorem is well-known [1-4]:

Theorem A Let $u(z)$ be a harmonic function in the upper half-plane $\mathbf{C}_{+}=\{z=x+i y=$ $\left.r \mathrm{e}^{\mathrm{i} \varphi}, y>0\right\}$ with continuous boundary values on the real axis. Suppose that

$$
u(z) \leq K r^{\rho}, \quad z \in \mathbf{C}_{+}, \quad r=|z| \geq 1, \quad \rho>1,
$$

and

$$
|u(z)| \leq K, \quad|z| \leq 1, \quad \operatorname{Im} z \geq 0
$$

Then

$$
u\left(r \mathrm{e}^{\mathrm{i} \varphi}\right) \geq-c K \frac{\left(1+r^{\rho}\right)}{\sin \varphi}, \quad r \mathrm{e}^{\mathrm{i} \varphi} \in \mathbf{C}_{+},
$$

where $c$ does not depend on $K, r, \varphi$ and the function $u(z)$.

Our objective is to establish the following theorem.

Theorem 1 Let $u(x)$ be a harmonic function in the half space $\mathbf{R}_{+}^{n}=\left\{x \in \mathbf{R}^{n}, x_{n}>0\right\}$ with continuous boundary values on the boundary $\partial \mathbf{R}_{+}^{n}$. Suppose that

$$
u(x) \leq K r^{\rho}, \quad x \in \mathbf{R}_{+}^{n}, \quad r=|x| \geq 1, \quad \rho>n-1,
$$

and

$$
|u(x)| \leq K, \quad|x| \leq 1, \quad x_{n} \geq 0 .
$$

Then

$$
u(x) \geq-c K \frac{\left(1+r^{\rho}\right)}{\sin ^{n-1} \varphi},
$$

where $c$ is a constant independent of $K, r, \varphi$ and the function $u(x)$.

Remark When $n=2$, the above theorem reduces to Theorem A. 


\section{Two Preliminary Lemmas}

The following Lemma 1 is a generalization of the Carleman's formula for harmonic functions in the half plane to the higher dimensional Euclidean half space.

Lemma 1 (Carleman's formula of harmonic functions in the half space) Let $u(x)$ be a harmonic function in $\mathbf{R}_{+}^{n}$ and continuous on $\overline{\mathbf{R}}_{+}^{n}$, and $\mathrm{d} \sigma(x)$ be the surface element of sphere in $\mathbf{R}_{+}^{n}$. Then, for $R>r>0$, we have

$$
\int_{\left\{x:|x|=R, x_{n}>0\right\}} u(x) \frac{n x_{n}}{R^{n+1}} \mathrm{~d} \sigma(x)+\int_{\left\{x: r<\left|x^{\prime}\right|<R, x_{n}=0\right\}} u\left(x^{\prime}\right)\left(\frac{1}{\left|x^{\prime}\right|^{n}}-\frac{1}{R^{n}}\right) \mathrm{d} x^{\prime}=A_{u}(r, R),
$$

where $A_{u}(r, R)=R^{-n} c_{1}(r)+c_{2}(r)$ is a function depending on $r$ and $R$. Here, functions $c_{1}(r), c_{2}(r)$ are defined respectively by

$$
\begin{gathered}
c_{1}(r)=\int_{\left\{x:|x|=R, x_{n}>0\right\}}\left(\frac{x_{n}}{u(x)}-x_{n} \frac{\partial u(x)}{\partial r}\right) \mathrm{d} \sigma(x), \\
c_{2}(r)=-\int_{\left\{x:|x|=r, x_{n}>0\right\}}\left(\frac{(n-1) x_{n}}{r^{n+1}}-x_{n} \frac{\partial u(x)}{\partial r}\right) \mathrm{d} \sigma(x)
\end{gathered}
$$

with $\frac{\partial u(x)}{\partial r}=\sum_{i=1}^{n} \frac{x_{i}}{r} \frac{\partial u}{\partial x_{i}}$.

Proof Applying the second Green's formula to the harmonic functions $u(x)$ and $v(x)=$ $\frac{x_{n}}{|x|^{n}}-\frac{x_{n}}{R^{n}}$ in the resulting sphere $B^{+}(r, R)=B(r, R) \cap \mathbf{R}_{+}^{n}$, we obtain

$$
\int_{\partial B^{+}(r, R)}\left(u(x) \frac{\partial v(x)}{\partial n}-v(x) \frac{\partial u(x)}{\partial n}\right) \mathrm{d} \sigma(x)=0,
$$

where $\partial / \partial n$ is the differentiation along the inward normal onto $B^{+}(r, R)$.

Since the function $v(x)$ is harmonic in $\mathbf{R}_{+}^{n} \backslash\{0\}$, the equations

$$
v(x)=0, \quad \frac{\partial v(x)}{\partial n}=\frac{n x_{n}}{R^{n+1}}, \quad x=\left(x^{\prime}, x_{n}\right)
$$

hold on the half sphere $\left\{x:|x|=R, x_{n} \geq 0\right\}$, and the equations

$$
v(x)=0, \quad \frac{\partial v(x)}{\partial n}=-\frac{|x|^{n}}{r}\left(\frac{n-1}{r^{n}}+\frac{1}{R^{n}}\right)
$$

hold on $\left\{x:|x|=r, x_{n}>0\right\}$. Moreover, the equations

$$
v(x)=0, \quad \frac{\partial v(x)}{\partial n}=\frac{1}{\left|x^{\prime}\right|^{n}}-\frac{1}{R^{n}}
$$

are true on $\left\{x=\left(x^{\prime}, 0\right): r<\left|x^{\prime}\right|<R\right\}$. Thus

$$
\begin{aligned}
0= & \int_{\partial B_{r, R}^{+}}\left(u(x) \frac{\partial v(x)}{\partial n}-v(x) \frac{\partial u(x)}{\partial n}\right) \mathrm{d} \sigma(x) \\
= & \int_{\left\{x \in \partial B_{r, R}^{+}, x_{n}>0\right\}}\left(u(x) \frac{\partial v(x)}{\partial n}-v(x) \frac{\partial u(x)}{\partial n}\right) \mathrm{d} \sigma(x)+\int_{\left\{x \in \partial B_{r, R}^{+}, x_{n}=0\right\}} u\left(x^{\prime}\right) \frac{\partial v\left(x^{\prime}\right)}{\partial n} \mathrm{~d} x^{\prime} \\
= & \int_{\left\{x:|x|=R ; x_{n}>0\right\}} u(x) \frac{n x_{n}}{R^{n+1}} \mathrm{~d} x^{\prime}+\int_{\left\{x: r<\left|x^{\prime}\right|<R, x_{n}=0\right\}} u\left(x^{\prime}\right)\left(\frac{1}{\left|x^{\prime}\right|^{n}}-\frac{1}{R^{n}}\right) \mathrm{d} x^{\prime} \\
& -\int_{\left\{x:|x|=r ; x_{n}>0\right\}} u(x) \frac{n x_{n}}{r^{n+1}} \mathrm{~d} x^{\prime}-\int_{\left\{x:\left|x^{\prime}\right|=r, x_{n}=0\right\}}\left(\frac{x_{n}}{|x|^{n}}-\frac{x_{n}}{R^{n}}\right) \frac{\partial u(x)}{\partial r} \mathrm{~d} \sigma(x) .
\end{aligned}
$$


The remainder term

$$
\begin{aligned}
& -\int_{\left\{x:|x|=r ; x_{n}>0\right\}} \frac{n x_{n}}{r^{n+1}} u(x) \mathrm{d} \sigma(x)-\int_{\left\{x: r<|x|<R, x_{n}=0\right\}}\left(\frac{x_{n}}{|x|^{n}}-\frac{x_{n}}{R^{n}}\right) \frac{\partial u(x)}{\partial r} \mathrm{~d} \sigma(x) \\
= & \frac{c_{1}(r)}{R^{n+1}}-c_{2}(r)
\end{aligned}
$$

is denoted by $A_{u}(r, R)$, a function depending on $r$ and $R$. This completes the proof of Lemma 1 .

Next, we will give a precise Nevanlinna's representation of harmonic functions in the half sphere by using Hörmander's theorem [5].

Lemma 2 (Nevanlinna's representation of harmonic functions in the half sphere) Let $u(x)$ be a harmonic function in $\mathbf{R}_{+}^{n}$. Then, on the closed half sphere $\bar{B}_{R}^{+}=\bar{B}_{R} \cap \mathbf{R}_{+}^{n}$ with $\bar{B}_{R}=\{x:|x|=R\}$, we have

$$
\begin{aligned}
u(x)= & \int_{\left\{y \in \mathbf{R}_{+}^{n}:|y|=R, y_{n}>0\right\}} \frac{R^{2}-|x|^{2}}{\omega_{n} R}\left(\frac{1}{|y-x|^{n}}-\frac{1}{\left|y-x^{*}\right|^{n}}\right) u(y) \mathrm{d} \sigma(y) \\
& +\frac{2 x_{n}}{\omega_{n}} \int_{\left\{y \in \mathbf{R}_{+}^{n}: r<\left|y^{\prime}\right|<R, y_{n}=0\right\}}\left(\frac{1}{\left|y^{\prime}-x\right|^{n}}-\frac{R^{n}}{|x|^{n}} \frac{1}{\left|y^{\prime}-\widetilde{x}\right|^{n}}\right) u\left(y^{\prime}\right) \mathrm{d} y^{\prime},
\end{aligned}
$$

where the reflection $\widetilde{x}$ of $x$ in $\partial B_{R}$ is defined by $\widetilde{x}=R^{2} x /|x|^{2}$, its direction corresponds with $x$, and $|x||\widetilde{x}|=R^{2}$.

Proof Note that $x \in \mathbf{R}^{n} \backslash\{0\}$, the inversion $x \rightarrow \widetilde{x}$ is the identity on $\partial B_{R}$. It is also an involution, that is, $(\widetilde{x})^{\widetilde{y}}=x$ for every $x \neq 0$. If $x, x^{\prime}$ and $y, y^{\prime}$ are two pairs of corresponding points, then the equation $|x|\left|x^{\prime}\right|=|y|\left|y^{\prime}\right|$ shows that the triangles with vertices $0, x, y$ and $0, x^{\prime}, y^{\prime}$ are similar, so that we may interchange $x$ and $x^{\prime}$ to obtain

$$
\begin{aligned}
& |y| /\left|x^{\prime}\right|=|x| /\left|y^{\prime}\right|=|x-y| /\left|x^{\prime}-y^{\prime}\right|, \\
& |y| /|x|=\left|x^{\prime}\right| /\left|y^{\prime}\right|=\left|x^{\prime}-y\right| /\left|x-y^{\prime}\right| .
\end{aligned}
$$

If $|y|=R$, then $y^{\prime}=y$ and (4) gives $|x-y| /\left|x^{\prime}-y\right|=\sqrt{|x| /\left|x^{\prime}\right|}=|x| / R$, which means that the sphere is harmonic with respect to $x$ and $x^{\prime}$.

Let

$$
E(x)=-\frac{|x|^{2-n}}{(n-2) \omega_{n}}, \quad n>2,
$$

where $\omega_{n}$ is the area of the unit sphere in $\mathbf{R}^{n}$. We know $E$ is locally integrable in $\mathbf{R}^{n}$. Now we define the following Green's function

$$
G_{R}(x, y)=E(x-y)-E\left((\widetilde{x}-y) \frac{|x|}{R}\right)=E(x-y)-E\left((x-\widetilde{y}) \frac{|y|}{R}\right)
$$

for $x, y \in \bar{B}_{R}$ and $x \neq y$, where the second equality follows from (5). The first expression shows that $G_{R}$ is a harmonic function of $y$ for fixed $x \neq y$, while the second expression shows that $G_{R}$ is a harmonic function of $x$ for fixed $y \neq x$. Clearly, we have

$$
G_{R}(x, y)=0 \quad \text { if } \quad|x|=R \quad \text { or } \quad|y|=R, \quad \text { and } \quad G_{R}(x, y) \leq 0 .
$$


For fixed $x$ with $|x|<R$ the inequality $|x-y|<|x|\left|x^{\prime}-y\right| / R$ is satisfied for all $y$ in $B_{R}$. Define a Green's function by

$$
G_{R}^{+}(x, y)=G_{R}\left(x, y^{*}\right)-G_{R}(x, y)=G_{R}(x, y)-G_{R}\left(x^{*}, y\right), \quad x, y \in B_{R}^{+}, \quad x \neq y
$$

for the half ball $B_{R}^{+}=B_{R} \cap \mathbf{R}_{+}^{n}$. Recall that $*$ denotes the reflection operation on the boundary plane $\partial \mathbf{R}_{+}^{n}$. It is clear that $G_{R}^{+}(x, y)=0$ if $x$ or $y$ is in $\partial \mathbf{R}_{+}^{n}$, and $G_{R}^{+}(x, y)-E(x-y)$ is harmonic in $x$ and $y$. We see that the Poisson kernel

$$
P_{R}^{+}(x, y)=\partial G_{R}^{+}(x, y) / \partial n, \quad x \in B_{R}^{+}, \quad y \in \partial B_{R}^{+}
$$

is positive, because $G_{R}^{+}(x, y) \rightarrow-\infty$ as $x \rightarrow y$, so that by the maximum principle $G_{R}^{+}(x, y)<0$ in $B_{R}^{+} \times B_{R}^{+}$. If $u(x)$ is harmonic near $\bar{R}_{R}^{+}$, Hörmander [2] proved that

$$
u(x)=\int_{\partial B_{R}^{+}} P_{R}^{+}(x, y) u(y) \mathrm{d} \sigma(y) .
$$

Below we provide an explict calculation for $P_{R}^{+}(x, y)$.

When $|y|=R$,

$$
\begin{array}{cc}
\frac{\partial G_{R}(x, y)}{\partial n}=\frac{\partial G_{R}(x, y)}{\partial y}=\frac{1}{\omega_{n} R} \frac{R^{2}-|x|^{2}}{|y-x|^{n}}, & |y|=R, \\
\frac{\partial G_{R}\left(x, y^{*}\right)}{\partial n}=\frac{\partial G_{R}\left(x, y^{*}\right)}{\partial y^{*}}=\frac{1}{\omega_{n} R} \frac{R^{2}-|x|^{2}}{\left|y^{*}-x\right|^{n}}, \quad\left|y^{*}\right|=R .
\end{array}
$$

So

$$
\begin{aligned}
P_{R}^{+}(x, y) & =\frac{\partial G_{R}^{+}(x, y)}{\partial n}=\frac{\partial G_{R}(x, y)}{\partial n}-\frac{\partial G_{R}\left(x^{*}, y\right)}{\partial n} \\
& =\frac{R^{2}-|x|^{2}}{\omega_{n} R}\left(\frac{1}{|y-x|^{n}}-\frac{1}{\left|y-x^{*}\right|^{n}}\right) .
\end{aligned}
$$

When $|y|<R, \quad y_{n}=0$,

$$
\begin{gathered}
\frac{\partial G_{R}(x, y)}{\partial n}=-\left.\frac{\partial G_{R}(x, y)}{\partial y_{n}}\right|_{y_{n}=0}=\frac{x_{n}}{\omega_{n}}\left[\frac{1}{|y-x|^{n}}-\frac{1}{|y-\widetilde{x}|^{n}} \frac{R^{n}}{|x|^{n}}\right], \\
\frac{\partial G_{R}\left(x^{*}, y\right)}{\partial n}=-\left.\frac{\partial G_{R}\left(x^{*}, y\right)}{\partial y_{n}}\right|_{y_{n}=0}=-\frac{x_{n}}{\omega_{n}}\left[\frac{1}{\left|y-x^{*}\right|^{n}}-\frac{1}{\left|y-\widetilde{x^{*}}\right|^{n}} \frac{R^{n}}{|x|^{n}}\right] .
\end{gathered}
$$

Hence,

$$
\begin{gathered}
P_{R}^{+}(x, y)=\frac{\partial G_{R}^{+}(x, y)}{\partial n}=\frac{\partial G_{R}(x, y)}{\partial n}-\frac{\partial G_{R}\left(x^{*}, y\right)}{\partial n} \\
=\frac{2 x_{n}}{\omega_{n}}\left(\frac{1}{|y-x|^{n}}-\frac{1}{|y-\widetilde{x}|^{n}} \frac{R^{n}}{|x|^{n}}\right), \\
u(x)=\int_{\left\{y \in \mathbf{R}_{+}^{n}:|y|=R, y_{n}>0\right\}} \frac{R^{2}-|x|^{2}}{\omega_{n} R}\left(\frac{1}{|y-x|^{n}}-\frac{1}{\left|y-x^{*}\right|^{n}}\right) u(y) \mathrm{d} \sigma(y) \\
+\frac{2 x_{n}}{\omega_{n}} \int_{\left\{y \in \mathbf{R}_{+}^{n}: r<\left|y^{\prime}\right|<R, y_{n}=0\right\}}\left(\frac{1}{\left|y^{\prime}-x\right|^{n}}-\frac{R^{n}}{|x|^{n}} \frac{1}{\left|y^{\prime}-\widetilde{x}\right|^{n}}\right) u\left(y^{\prime}\right) \mathrm{d} y^{\prime} .
\end{gathered}
$$

The proof of Lemma 2 is completed. 


\section{Proof of Theorem 1}

We apply Lemma 1 to the harmonic function $u(x)$ to obtain

$$
\begin{aligned}
& \frac{n}{R^{n+1}} \int_{\left\{x:|x|=R, x_{n}>0\right\}} x_{n} u^{-}(x) \mathrm{d} \sigma(x) \\
& +\int_{\left\{x: r<\left|x^{\prime}\right|<R, x_{n}=0\right\}} u^{-}\left(x^{\prime}\right)\left(\frac{1}{\left|x^{\prime}\right|^{n}}-\frac{1}{R^{n}}\right) \mathrm{d} x^{\prime}+A_{u}(r, R) \\
= & \frac{n}{R^{n+1}} \int_{\left\{x:|x|=R, x_{n}>0\right\}} x_{n} u^{+}(x) \mathrm{d} \sigma(x)+\int_{\left\{x: r<\left|x^{\prime}\right|<R, x_{n}=0\right\}} u^{+}\left(x^{\prime}\right)\left(\frac{1}{\left|x^{\prime}\right|^{n}}-\frac{1}{R^{n}}\right) \mathrm{d} x^{\prime} .
\end{aligned}
$$

Here and in the sequel, $u^{-}=(-u)^{+}$, i.e., $u=u^{+}-u^{-}$, and the remainder term is

$$
A_{u}(r, R)=\frac{c_{1}(r)}{R^{n+1}}-c_{2}(r)
$$

in which $c_{1}, c_{2}$ are functions depending only on $r$.

The terms on the right-hand side of (6) can be estimated by using (1):

$$
\begin{gathered}
\frac{n}{R^{n+1}} \int_{\left\{x:|x|=R, x_{n}>0\right\}} x_{n} u^{+}(x) \mathrm{d} \sigma(x) \leq n \omega_{n} K R^{\rho-1}, \\
\int_{\left\{x: r<\left|x^{\prime}\right|<R, x_{n}=0\right\}} u^{+}\left(x^{\prime}\right)\left(\frac{1}{\left|x^{\prime}\right|^{n}}-\frac{1}{R^{n}}\right) \mathrm{d} x^{\prime} \leq \frac{n \omega_{n-1} K R^{\rho-1}}{(\rho-1)(\rho+n-1)} .
\end{gathered}
$$

Thus, for $R \geq 2$ and (6), we can obtain

$$
\begin{gathered}
\frac{n}{R^{n+1}} \int_{\left\{x:|x|=R, x_{n}>0\right\}} x_{n} u^{-}(x) \mathrm{d} \sigma(x) \leq c R^{\rho-1}, \\
\int_{\left\{x: r<\left|x^{\prime}\right|<R, x_{n}=0\right\}} \frac{u^{-}\left(x^{\prime}\right)}{\left|x^{\prime}\right|^{n}} \mathrm{~d} x^{\prime} \\
\leq \frac{2^{n}}{2^{n}-1} \int_{\left\{x: r<\left|x^{\prime}\right|<R, x_{n}=0\right\}} u^{-}\left(x^{\prime}\right)\left(\frac{1}{\left|x^{\prime}\right|^{n}}-\frac{1}{(2 R)^{n}}\right) \mathrm{d} x^{\prime} \leq c R^{\rho-1},
\end{gathered}
$$

where $c$ is a constant independent of the variables.

Lemma 2 and estimates (7) and (8) allow us to find a lower bound for the function $u(x)$. We have

$$
\begin{aligned}
-u(x)= & \frac{R^{2}-|x|^{2}}{\omega_{n} R} \int_{\left\{y:|y|=R, y_{n}>0\right\}}\left(\frac{1}{|y-x|^{n}}-\frac{1}{\left|y-x^{*}\right|^{n}}\right)(-u(y)) \mathrm{d} \sigma(y) \\
& +\frac{2 x_{n}}{\omega_{n}} \int_{\left\{y: r<\left|y^{\prime}\right|<R, y_{n}=0\right\}}\left(\frac{1}{\left|y^{\prime}-x\right|^{n}}-\frac{R^{n}}{|x|^{n}} \frac{1}{\left|y^{\prime}-\widetilde{x}\right|^{n}}\right)\left(-u\left(y^{\prime}\right)\right) \mathrm{d} y^{\prime} .
\end{aligned}
$$

Both integral kernels are positive, since they are derivatives of the Green function with respect to the outward normal. This permits us to replace $-u$ by $u^{-}$in the integrals and simultaneously to transform identity (9) into the inequality. In the following we will estimate the kernels appearing in the integrals. 
Let $r=|x| \geq 2, R=2 r$. Then, by the mean value theorem for derivatives, we obtain the estimate of the kernel in the first integral

$$
\begin{aligned}
& \frac{R^{2}-|x|^{2}}{\omega_{n} R}\left(\frac{1}{|y-x|^{n}}-\frac{1}{\left|y-x^{*}\right|^{n}}\right) \\
= & \frac{R^{2}-r^{2}}{\omega_{n} R}\left(\frac{1}{\left|R^{2}-2 R r \cos (\theta-\varphi)+r^{2}\right|^{n / 2}}-\frac{1}{\left|R^{2}-2 R r \cos (\theta+\varphi)+r^{2}\right|^{n / 2}}\right) \\
\leq & \frac{R^{2}-r^{2}}{\omega_{n} R} \frac{2 R r \sin \theta \sin \varphi}{(R-r)^{n+2}} \leq \frac{c \sin \theta}{R^{n-1}},
\end{aligned}
$$

where $x_{n}=|x| \cos \theta, y_{n}=|y| \cos \varphi$, and $c$ is a constant depending only on $n$. So

$$
\begin{aligned}
& \int_{\left\{y:|y|=R, y_{n}>0\right\}} \frac{R^{2}-|x|^{2}}{\omega_{n} R}\left(\frac{1}{|y-x|^{n}}-\frac{1}{\left|y-x^{*}\right|^{n}}\right)(-u(y)) \mathrm{d} \sigma(y) \\
\leq & \int_{\left\{y:|y|=R, y_{n}>0\right\}} \frac{R^{2}-|x|^{2}}{\omega_{n} R} \leq\left(\frac{1}{|y-x|^{n}}-\frac{1}{\left|y-x^{*}\right|^{n}}\right) u^{-}(y) \mathrm{d} \sigma(y) \\
\leq & \frac{c}{R^{n}} \int_{\left\{y:|y|=R, y_{n}>0\right\}} u^{-}(y) y_{n} \mathrm{~d} \sigma(y) \leq c R^{\rho},
\end{aligned}
$$

where $c$ is a constant independent of the variables.

Using the inequalities

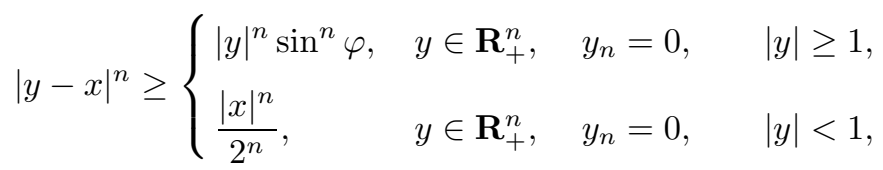

and setting $r=|x| \geq 2, R=2 r$, we obtain the estimate of the kernel in the second integral:

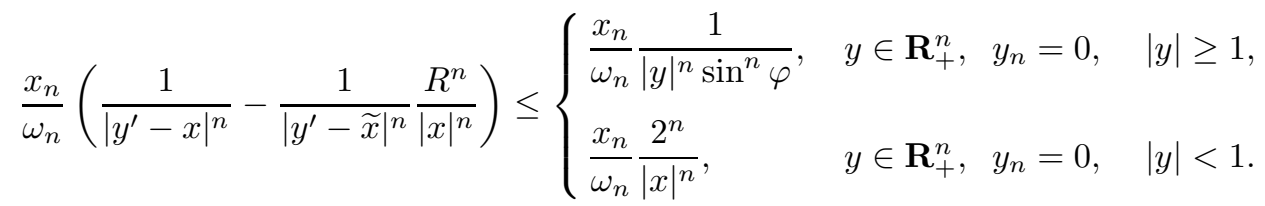

For the second integral, we have, for $|y| \geq 1$,

$$
\begin{aligned}
& \int_{\left\{y: r<\left|y^{\prime}\right|<R, y_{n}=0\right\}} \frac{2 x_{n}}{\omega_{n}}\left(\frac{1}{\left|y^{\prime}-x\right|^{n}}-\frac{1}{\left|y^{\prime}-\widetilde{x}\right|^{n}} \frac{R^{n}}{|x|^{n}}\right)\left(-u\left(y^{\prime}\right)\right) \mathrm{d} y^{\prime} \\
= & \int_{\left\{y: r<\left|y^{\prime}\right|<R, y_{n}=0\right\}} \frac{2 x_{n}}{\omega_{n}}\left(\frac{1}{\left|y^{\prime}-x\right|^{n}}-\frac{1}{\left|y^{\prime}-\widetilde{x}\right|^{n}} \frac{R^{n}}{|x|^{n}}\right) u^{-}\left(y^{\prime}\right) \mathrm{d} y^{\prime} \\
\leq & c K \frac{1+R^{\rho}}{\sin ^{n-1} \varphi} .
\end{aligned}
$$

Substituting inequalities (10) and (11) into (9), we obtain

$$
-u(x) \leq A R^{\rho}+c K \frac{1+r^{\rho}}{\sin ^{n-1} \varphi}+\frac{2^{n}}{\omega_{n} r^{n-1}} \int_{-1}^{1}\left(u^{-}(t)+u^{-}(-t)\right) \mathrm{d} t .
$$

By using (2), the third integral is bounded uniformly with respect to $x^{\prime}$.

Therefore, for $|x| \geq 2$, we get

$$
u(x) \geq-c K \frac{1+r^{\rho}}{\sin ^{n-1} \varphi} .
$$

Thus Theorem 1 is proved. 


\section{References}

[1] Levin B Y. Lectures on Entire Functions. Transl Math Monographs, Vol 150. Providence, RI: Amer Math Soc, 1996

[2] Krasichkov-Ternovskii I F. An estimate for the subharmonic difference of subharmonic functions I. Mat Sb, 1977, 102(2): 216-247; II. Mat Sb, 1977, 103(1): 69-111; English Transl in Math USSR-Sb, 1977, 32 191-218

[3] Nikol'skiǐ N K. Selected Problems of the Weighted Approximation and of Spectral Analysis. Trudy Mat Inst Steklov Inst Steklov, 1974, 120; English transl in Proc Steklov Inst Math, 1976, 120

[4] Matsaev V I, Mogulskiǐ E Z, Adivision theorem for analytic functions with a given majorant, and some of its applications. Zap Nauchn Sem Leningrad Otdel Mat Inst Steklov (LOMI), 1976, 56: 73-89; English transl in J Soviet Math, 1980, 56

[5] Hörmander L. Notions of Convexity. Boston, Basel, Berlin: Birkhäuser, 1994

[6] Stein E M. Harmonic Analysis. Princeton, NJ: Princeton University Press, 1993

[7] Axler S, Bourdon P, Ramey W. Harmonic Function Theory Second Edition. New York: Springer-Verlag, 1992

[8] Gilbarg D, Trudinger N S. Elliptic Partial Differential Equations of Second Order. Berlin: Springer-Verlag, 2001

[9] Stein E M, Weiss G. Introduction to Fourier Analysis on Euclidean Space. Princeton, NJ: Princeton Univ Press, 1971

[10] Deng G T. On zeros of analytic functions in half Plane. Acta Math Sci, 2006, 26A(1): 45-48 